EPJ Web of Conferences 81, 01020 (2014)

DOI: $10.1051 /$ epjconf/ 20148101020

(C) Owned by the authors, published by EDP Sciences, 2014

\title{
Search for Dark Photons with Accelerators
}

\author{
Harald Merkel ${ }^{1, a}$ \\ for the A1 Collaboration \\ ${ }^{1}$ Institut für Kernphysik, Johannes Gutenberg-Universität Mainz, 55099 Mainz, Germany
}

\begin{abstract}
A dark photon as the mediator of an interaction of the dark sector is a well motivated extension of the standard model. While possible dark matter particles are heavy and seem to be beyond the reach of current accelerators, the dark photon is not necessarily heavy and might have a mass in the range of existing accelerators. In recent years, an extensive experimental program at several accelerators for the search for dark photons were established. In this talk, recent results and progress in the determination of exclusion limits with accelerators is presented.
\end{abstract}

\section{Introduction}

The clear gravitational evidence for dark matter is one of the most important puzzles of today's physics. In recent years, the lightest super-symmetric particle was one of the most promising candidates for an explanation of dark matter with particle physics concepts. Meanwhile, since no hints for super-symmetry were discovered up to now at the LHC experiments, and no direct scattering experiments showed clear results, it is necessary to extend the search for dark matter to more general concepts.

Assuming, that dark matter can be described in a particle scheme similar to the standard model, it is reasonable to expect not only a single dark matter particle but a whole dark sector of particles with corresponding symmetries and interactions. Therefore one should not restrict the search to the dark matter particles themselves, but can extend the search to the interaction of the dark sector. The simplest case of an interaction is given by an massive U(1) gauge boson, sometimes called the "dark photon" $\gamma^{*}$ (sometimes also denoted as $A$ or $U$ boson).

Such a gauge boson of the dark sector has not to be heavy, but the coupling to standard model matter is very weak. A straight forward formalism for an residual interaction with SM matter is given by the kinetic mixing of the dark photon field with the electromagnetic field [1], described by a mixing parameter $\epsilon$. From general considerations [2] one expects a mixing parameter in the range of $10^{-3}<\epsilon<10^{-6}$.

Besides the theoretical motivation of such a dark photon, several experimental puzzles might be solved by the existence of a dark photon. The cosmic positron excess seen by PAMELA, Fermi-LAT and recently AMS-02 could be explained by dark matter annihilation into dark matter (other sources, like e.g. quasars, are also not yet excluded, of course). Also the discrepancy between the measurement of $g-2$ of the muon could be explained by a dark photon exchange in a loop graph [3-5].

\footnotetext{
ae-mail: merkel@kph.uni-mainz.de
} 

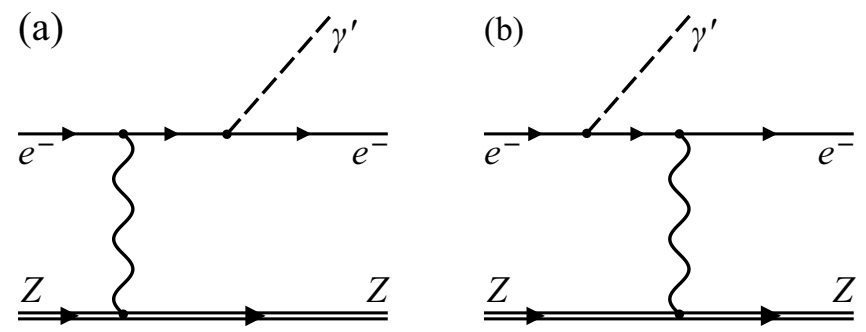

Figure 1. Radiative production of a dark photon via Bremsstrahlung of a heavy target nucleus.

All the experimental hints favour a mass range of such a dark photon in the range below a few $\mathrm{GeV}$. This energy range is accessible by several accelerators. in this talk the current program for dark photon searches at accelerators will be discussed.

\section{Dark Photon Searches at fixed Target Experiments}

Bjorken et al.[6] first calculated in Weizsäcker-Williams approximation the cross section of dark photon production via electron scattering from a heavy target. Fig. 1 shows the corresponding graphs for radiative production from outgoing (a) and incoming (b) electron lines.

The dark photon can decay into a lepton pair. The coupling of the dark photon is so small, that the live time of the dark photon is long enough for a few micrometer until several meters of decay length. The experimental technique for searching a dark photon in a fixed target experiment is to just look for a lepton pair at the mass of the dark photon.

Unfortunately, a lepton pair can also be produced in a pure QED process via a virtual photon, giving a huge background to the signal process. Therefore the experimental challenge is to identify a peak in the mass of the lepton pair at the mass of the dark photon on top of large, but smooth background of radiative lepton pairs. This background can be calculated accurately [7].

Since the peak width is given by the experimental resolution, high resolution spectrometers are well suited for this experiments. First test experiments were performed by the A1 collaboration at MAMI [8] and by the APEX collaboration at Jefferson Laboratory [9].

After these first test measurements, an extended measurement was performed at MAMI [10]. Several beam energies were used to cover the mass range from $50 \mathrm{MeV} / c^{2}$ up to $300 \mathrm{MeV} / c^{2}$. No signal of a dark photon was found. Fig. 2 shows the exclusion limits derived from this experiment as yellow shaded region, together with the limits by the APEX test experiment.

For this figure, all settings were added up to derive a single exclusion limit. Also include in this picture are the areas excluded by the measurement of $g-2$ of the electron and the muon. The area denoted as E774 shows exclusion limits of a beam dump experiment. The areas denoted by WASA, KLOE-2, BaBar and HADES are discussed in the next section.

The red line and a dashed $2 \sigma$ error band shows the region of the parameter space, where the $(g-2)$ anomaly of the muon could be explained by the existence of a dark photon. As can be seen, this region is now nearly completely excluded by the recent experiments.

\section{Search for Dark Photons in Meson Decays}

While fixed target experiments make use of the high luminosity achievable in modern experiments (more than $Z^{2} L=10^{39} \mathrm{~s}^{-1} \mathrm{~cm}^{-2}$ ) for dedicated experiments, collider experiments have already accumulated large data samples. A dark photon can in principle occur in all processes involving the 


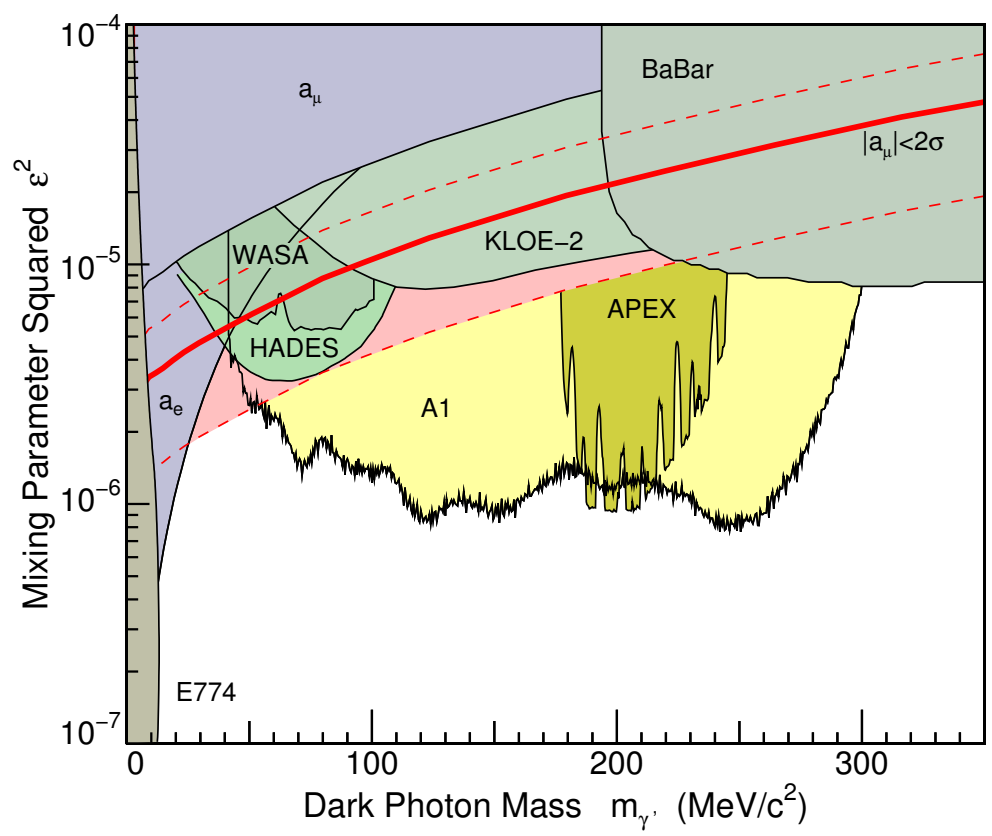

Figure 2. Exclusion limit derived by the A1 experiment. See text for the explanation of the different lines.

production of a lepton pair via a virtual photon. Fig. 3 shows the decay of pion into a real and a virtual photon, which decays into a lepton pair.

Again, the signature of a dark photon would be a peak in the mass spectrum of the lepton pair. While the resolution of collider experiments is much lower than the resolution of spectrometers, the background is also lower, allowing to extract sensitive results from meson decays. Table 1 shows the channels analyzed by the BaBar, KLOE-2, WASA, and HADES collaboration, the corresponding exclusion limits are included in fig. 2.

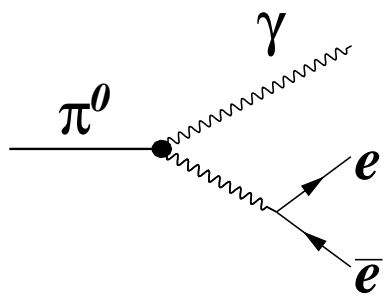

Figure 3. A dark photon can appear in all meson decays with a lepton pair.

\section{Conclusions and Outlook}

The dark photon is well motivated extension of the standard model and searches started at several laboratories. From the analysis of collider data already significant exclusion limits could be extracted. 
Table 1. Please write your table caption here

\begin{tabular}{lll}
\hline Collaboration & Reaction & Reference \\
\hline BaBar & $e^{+} e^{-} \rightarrow \Upsilon \rightarrow \mu^{+} \mu^{-} \gamma$ & {$[11]$} \\
KLOE-2 & $\phi \rightarrow e^{+} e^{-} \gamma$ & {$[12]$} \\
WASA & $\pi^{0} \rightarrow e^{+} e^{-} \gamma$ & {$[13]$} \\
HADES & $p+p, p+N b, A r+K C l \rightarrow e^{+}+e^{-}$ & {$[14]$} \\
KLOE-2 & $e^{+} e^{-} \rightarrow \mu^{+} \mu^{-} \gamma$ & {$[15]$} \\
\hline
\end{tabular}

Together with fixed target experiments, the region of the $(g-2)$ of the muon could be excluded nearly completely.

Future experiments will concentrate on the region of smaller mixing parameter $\epsilon$. Several experiments plan to use for this the secondary vertex of the dark photon decay. By demanding a secondary vertex the background by production of the lepton pair via a virtual photon can be suppressed completely.

In Mainz a new accelerator (MESA) will be constructed, which consists of a super-conduction energy-recovery linac at low energies. It is planned to equip this accelerator with a pair of high resolution spectrometers, which would be ideal to determine limits in the mass region below $50 \mathrm{MeV} / \mathrm{c}^{2}$, which cannot be reached by current setups.

\section{References}

[1] B. Holdom, Phys. Lett. B 166, 196 (1986).

[2] N. Arkani-Hamed et al., Phys. Rev. D 79, 015014 (2009).

[3] G. W. Bennet et al., Phys. Rev. D 73, 072003 (2006).

[4] M. Pospelov, Phys. Rev. D 80, 095002 (2009).

[5] P. Fayet, Phys. Rev. D 75, 115017 (2007).

[6] J. D. Bjorken et al., Phys. Rev. D 80, 075018 (2009).

[7] T. Beranek, H. Merkel and M. Vanderhaeghen, Phys. Rev. D 88, 015032 (2013).

[8] H. Merkel et al. (A1 Collaboration), Phys. Rev. Lett. 106, 251802 (2011).

[9] S. Abrahamyan et al. (APEX Collaboration), Phys. Rev. Lett. 107, 191804 (2011).

[10] H. Merkel et al., Phys. Rev. Lett. 112, 221802 (2014).

[11] B. Aubert et al., Phys. Rev. Lett. 103, 081803 (2009).

[12] D. Babusci et al., Phys. Lett. B 720, 111 (2013).

[13] P. Adlarson et al., Phys. Lett. B 726, 187 (2013).

[14] G. Agakishiev et al., Phys. Lett. B 731, 265 (2014).

[15] D. Babusci et al., arXiv:1404.7772 (2014). 